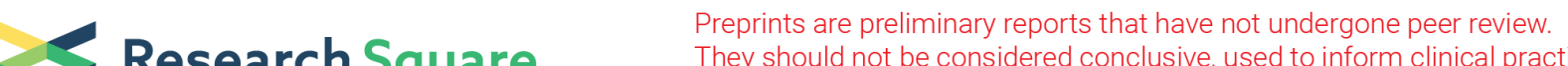 Research Square They should not be considered conclusive, used to inform clinical practice, or referenced by the media as validated information.
}

\section{Noninvasive Chromosome Screening for Evaluating the Clinical Outcomes of Patients With Recurrent Pregnancy Loss or Repeated Implantation Failure}

Haitao Xi

Wenzhou Medical University

Lin Qiu

The Second Affiliated Hospital and Yuying Children's Hospital of Wenzhou Medical University

Yaxin Yao

Yikon Genomics

Lanzi Luo

Wenzhou Medical University

Liucai Sui

The Second Affiliated Hospital and Yuying Children's Hospital of Wenzhou Medical University

\section{Yanghua Fu}

The Second Affiliated Hospital and Yuying Children's Hospital of Wenzhou Medical University

\section{Qiuyi Weng}

The Second Affiliated Hospital and Yuying Children's Hospital of Wenzhou Medical University

\section{Jing Wang}

Yikon Genomics

Junzhao Zhao

The Second Affiliated Hospital and Yuying Children's Hospital of Wenzhou Medical University

Yingzheng Zhao ( $\square$ pharmtds@163.com )

Wenzhou Medical University

\section{Research Article}

Keywords: Noninvasive chromosome screening, repeated implantation failure, recurrent pregnancy loss, assisted reproductive technology, clinical outcomes

Posted Date: December 15th, 2021

DOI: https://doi.org/10.21203/rs.3.rs-1070159/v1

License: (c) (1) This work is licensed under a Creative Commons Attribution 4.0 International License.

Read Full License 


\section{Abstract}

Background: This retrospective cohort study determines whether noninvasive chromosome screening (NICS) for aneuploidy can improve the clinical outcomes of patients with recurrent pregnancy loss (RPL) or repeated implantation failure (RIF) in assisted reproductive technology.

Methods: A total of 273 women with a history of RPL or RIF between 2018 and 2021 were included in this study. We collected data of all oocyte retrieval cycles and single blastocyst resuscitation transfer cycles.

Results: For the RPL patients, NICS reduced the miscarriages rate per frozen embryo transfer (FET), improved the ongoing pregnancies rate and live birth rate: $17.9 \%$ vs $42.6 \%$, adjusted $0 \mathrm{R} 0.39,95 \% \mathrm{Cl}$ $0.16-0.95 ; 40.7 \%$ vs $25.0 \%$, adjusted OR $2.00,95 \% \mathrm{Cl} 1.04-3.82 ; 38.9 \%$ vs $20.6 \%$, adjusted OR 2.53 , $95 \%$ $\mathrm{Cl} 1.28-5.02$, respectively. For the RIF patients, the pregnancy rates per FET in the NICS group were significantly higher than in the non-NICS group ( $46.9 \%$ vs. $28.7 \%$, adjusted OR $2.82,95 \% \mathrm{Cl} 1.20-6.66$ ).

Conclusions: This study demonstrated that selection of euploid embryos through NICS can reduce the miscarriage rate of patients with RPL and improve the clinical pregnancy rate of patients with RIF. Our data suggested that NICS could be used as a diagnostic test in clinical practice.

\section{Background}

In vitro fertilization-embryo transfer (IVF-ET) is an effective method to treat infertility widely performed worldwide [1]. Nevertheless, chromosomal abnormalities often exist in early human embryos, leading to embryo implantation failure and pregnancy loss during IVF treatment [2,3], especially in patients with a history of recurrent pregnancy loss (RPL) and repeated implantation failure (RIF) [4-6]. RPL is defined as the loss of $\geq 2$ pregnancies [7], whereas RIF as the failure of $\geq 3$ implantations. Trilochan et al. [8] reported that chromosome abnormalities were detected in 3,975 of 7,396 (53.7\%) cases of miscarriage tissues. Besides, Kort et al. [6] analyzed 10,711 cases of blastocysts retrospectively and found that the incidence of embryo aneuploidy was significantly higher in patients with RIF than in the control group.

Currently, the most commonly used embryo selection method is morphological evaluation. However, the chromosomes of embryos cannot be identified via morphology [2, 3]. Among blastocysts with good morphology, only $42 \%$ of embryos have completely normal chromosomes. Among them, only $30 \%$ of ICM graded A Embryos, the chromosomes are normal[9]. Therefore, in some special populations (such as women with recurrent miscarriage and repeated implantation failure patients), embryos are usually evaluated through the pre-implantation genetic testing for aneuploidy (PGT-A) [10-12]. A retrospective study showed that the live birth and clinical pregnancy rate were improved through the PGT-A in women with RPL[13]. The authors included 1,389 blastocysts derived from PGT-A cycles in IVF patients with advanced maternal age, those with repeated implantation failure, those with recurrent miscarriage, and oocyte donors. Compared the control group without PGT-A, the live birth rates of these four groups are both improved[14]. However, specific equipment and extensive expertise are required for the biopsy procedure, restraining the utility of PGT-A in assisted reproduction. Moreover, the embryos are screened 
for chromosomal ploidy before transferring to the uterus $[15,16]$, and the long-term impact of biopsy is an important concern for undetermined health risks, such as adrenal development and response to cold stress [17], epigenetic reprogramming [18], and neurological conditions [19]. Notably, implantation rates are lower with increased biopsied trophectoderm (TE) cell numbers and sizes than with appropriate cell numbers and sizes $[20,21]$.

Stigliani et al. first observed genomic DNA contents in embryo culture medium, [22]. Since then, multiple studies have been published using culture medium or blastocoelic fluid for analyzing chromosomal ploidy [23-30]. Xu et al. first reported a noninvasive chromosome screening (NICS) assay based on a Multiple Annealing and Looping-Based Amplification Cycle-Next Generation Sequencing (NGS) strategy using spent blastocyst culture medium, which was validated in 42 IVF and resulted in five live births among seven women [26]. Fang et al. obtained an ongoing pregnancy rate of $58 \%$ and reported 27 normal live births in a pilot clinical study using NICS [28]. Nevertheless, the clinical application of NICS has been evaluated only in small-scale trials.

In this study, we designed a retrospective cohort study including 273 patients with RPL or RIF to confirm the clinical value of NICS. To the best of our knowledge, this is the first large-scale validation study of NICS in the RPL or RIF patients.

\section{Methods}

\section{Study Participants and Data Collection}

We initially included 303 women with a history of RPL ( $\geq 2$ events) or RIF ( $\geq 3$ events) from July 2019 to May 2021, according to the records of the Reproductive Centre at the Second Affiliated Hospital of Wenzhou Medical University. However, ten women that abandoned embryo transfer and 20 women with chromosomal rearrangements, abnormal uterine cavity morphology, endometrial lesions, endometrial injury, intrauterine effusion, or untreated hydrosalpinx were excluded from the study. The final cohort comprised 273 women, from which we collected data of all oocyte retrieval cycles and single blastocyst resuscitation transfer cycles.

The variables analyzed in the study were: age at retrieval, the history of pregnancies, live births, and miscarriages, body mass index (BMI), hormone levels, the number of oocyte retrieval cycles, oocytes, cleavages, and D5 or D6 blastocysts, and blastocyst morphology (expansion, inner cell mass, and trophectoderm). Patients with RPL or RIF received the noninvasive chromosome screening for aneuploidy were included in the NICS group, while those underwent conventional morphology embryo transfer during the same period were included in the non-NICS group. The euploid embryos were transferred in the NICS group. The study design is illustrated in Figure 1.

\section{Oocyte Retrieval and Embryo Culture}

This study was performed at the first IVF/Intracytoplasmic Sperm Injection (ICSI) cycles after injection of $3.75 \mathrm{mg}$ triptorelin for prolonged pituitary downregulation in the follicular phase of the menstrual cycle. 
Ovarian stimulation with exogenous gonadotropins promoted the growth of follicles. When two or more leading follicles reached $18 \mathrm{~mm}$, ovulation was induced with 10,000 IU human chorionic gonadotropin (hCG). Oocyte retrieval was performed at $35 \mathrm{~h}$ post-hCG administration. Cumulus-enclosed oocytes were separated from the follicular fluid, placed in medium, and incubated at $37^{\circ} \mathrm{C}$ incubated d Cumulus-encl 2 atmosphere for $2 \mathrm{~h}$. Routine IVF or ICSI was performed based on sperm quality. The embryos were placed in droplets of G-1 PLUS medium (Vitrolife, Göteborg Sweden) in AMP-30D incubators (Bioz, Los Altos, CA, USA) in a $6.0 \% \mathrm{CO}_{2}$ and $5 \% \mathrm{O}_{2}$ balance $\mathrm{N}_{2}$ atmosphere at $37^{\circ} \mathrm{C}$.

\section{Blastocyst Culture and Transfer}

D3 embryos were placed in 30- $\mu$ L droplets of G-2 PLUS medium (Vitrolife) supplemented with washed and pre-gassed mineral oil (Sage, Atlanta, GA, USA) and cultured to the blastocyst stage in AMP-30D incubators (Bioz) in a $6.0 \% \mathrm{CO}_{2}$ and $5 \% \mathrm{O}_{2}$ balance $\mathrm{N}_{2}$ atmosphere at $37^{\circ} \mathrm{C}$. At $2 \mathrm{~d}$ of culture, the development and quality of blastocysts were evaluated according to the blastocyst scoring system, including expansion (1-6), inner cell mass (A, B, C), and trophectoderm $(A, B, C)$. Blastocysts were categorized into good, fair, or poor quality based on the simplified SART embryo scoring system[29]. The grade is good when the ICM/TE is $A A, A B$ or $B A$; the grade is fair when the ICM/TE is $B B$ or $A C$; the grade is poor when the ICM/TE is $C A, B C$ or CB. All blastocysts were previously frozen and thawed by vitrification using the Cryotop Safety kit (Kitazato, Fuji-shi, Shizuoka, Japan), according to the manufacturer's instructions. A single blastocyst was selected for transfer to each patient based on morphology (nonintervention group) or NICS results (intervention group).

\section{Sample Collection for NICS Assay}

To prevent medium cross-contamination, different Pasteur pipettes were used for each embryo. Approximately $30 \mu \mathrm{L}$ of blastocyst medium from each embryo was transferred into RNase-DNase-free PCR tubes containing $5 \mu \mathrm{L}$ of cell lysis buffer (Yikon Genomics, Suzhou, China). We also collected the same amount of blastocyst culture medium to be used as a negative control. All collected samples were flash-frozen in liquid nitrogen and stored at $-80^{\circ} \mathrm{C}$ until subjected to the NICS assay.

\section{Whole-genome amplification (WGA) and NGS Data Analysis}

Whole-genome amplification was performed using culture media, followed by library preparation using ChromInst (Yikon Genomics; EK100100724 NICS Inst Library Preparation Kit). NGS was performed on an Illumina MiSeq platform that yielded approximately 2 million sequence reads from each sample. The high-quality reads were extracted and mapped to the human hg19 genome. After removing duplication reads, the read numbers were counted along the whole genome with a bin size of $1 \mathrm{Mb}$ and normalized by the GC content as well as a reference dataset to represent the relative copy number. The copy number of each bin was then segmented by circular binary segmentation (CBS) algorithms to merge bins with similar trends and calculate the final copy numbers.

\section{Assessment of Outcome Variables}


Our primary outcomes included the clinical pregnancy rates, miscarriage rates, and ongoing pregnancy rates. The clinical pregnancy rate was defined as the number of cycles with gestational sacs visualized on transvaginal ultrasonography divided by the total number of transplant cycles. The miscarriage rate was calculated as the number of pregnancy failures after a gestational sac was documented by transvaginal ultrasonography divided by the total number of clinical pregnancies. The ongoing pregnancy rate was the number of cycles of any pregnancy that went beyond 12 weeks of gestation divided by the total number of transplant cycles. Ectopic pregnancy was considered a clinical pregnancy and not a miscarriage.

\section{Statistical Analysis}

Descriptive data for continuous variables are presented as means \pm standard deviation, whereas categoric data as numbers and percentages. The Student's $t$-test or Mann-Whitney test were used for identifying significant differences in parametric and non-parametric data, respectively. The chi-square test or Fisher exact test were used for categoric variables in each group. Multiple logistic regression analysis was conducted to compare the outcomes of NICS and non-NICS groups after controlling the covariables at $p<0.10$ and covariables considered clinically influential.

All analyses were carried out using the statistical software BMI SPSS, Version 25. A P value $<0.05$ was considered to denote statistical significance.

\section{Results}

\section{Variable Analysis}

Of the 303 women initially included in the study, 10 that abandoned embryo transfer and 20 that had no transferrable embryos were excluded from the final cohort. Finally, blastocysts were transferred to 273 women during 407 transfer cycles (Fig. 1).

Of the 173 patients with a history of RPL, were enrolled in the study, of which 84 were for the NICS group and 89 were included as the non-NICS group. In total, 113 FET cycles were performed for the patients in the NICS group, and 136 high-quality blastocysts were obtained from 89 patients (Table 1). Of the 100 patients with a history of RIF, 44 were for the NICS group and 56 for the non-NICS group. In total, 64 FET cycles were performed for the patients in the NICS group, and 94 high-quality blastocysts were obtained from the non-NICS group (Table 2).

Table 1. Baseline demographics of patients with recurrent pregnancy loss (RPL) after embryo selection by non-invasive chromosome screening (NICS) versus blastocyst morphology (Non-NICS). 


\begin{tabular}{|c|c|c|c|}
\hline Variable & NICS & Non-NICS & $p$-value \\
\hline Number of patients & 84 & 89 & \\
\hline Female age $(y)$ & $32.4 \pm 3.8$ & $33.4 \pm 4.7$ & 0.109 \\
\hline Female body mass index $\left(\mathrm{kg} \mathrm{m}^{-2}\right)$ & $22.1 \pm 2.8$ & $22.4 \pm 3.1$ & 0.371 \\
\hline Male age $(y)$ & $34.7 \pm 4.1$ & $35.2 \pm 4.7$ & 0.381 \\
\hline Male body mass index $\left(\mathrm{kg} \mathrm{m}^{-2}\right)$ & $24.0 \pm 3.0$ & $24.5 \pm 3.2$ & 0.293 \\
\hline Infertility duration (y) & $1.4 \pm 0.7$ & $2.1 \pm 2.2$ & 0.001 \\
\hline Number of prior miscarriages & $2.9 \pm 1.3$ & $2.5 \pm 1.1$ & 0.023 \\
\hline Number of previous embryo transfers & $0.63 \pm 1.0$ & $2.2 \pm 1.8$ & $<0.001$ \\
\hline Number of prior live births & $5(6.0 \%)$ & $1(1.1 \%)$ & 0.108 \\
\hline Prolactine $\left(\mathrm{ng} \mathrm{m}^{-1}\right)$ & $20.0 \pm 44.9$ & $21.4 \pm 45.9$ & 0.831 \\
\hline Testosterone & $0.5 \pm 0.3$ & $0.9 \pm 4.3$ & 0.415 \\
\hline Progesterone & $0.7 \pm 0.5$ & $0.6 \pm 0.6$ & 0.357 \\
\hline Follicle-stimulating hormone & $6.8 \pm 2.1$ & $7.5 \pm 4.2$ & 0.158 \\
\hline Luteinizing hormone & $4.8 \pm 3.7$ & $5.8 \pm 9.6$ & 0.349 \\
\hline Estradiol & $62.7 \pm 58.1$ & $66.7 \pm 71.88$ & 0.673 \\
\hline Number of $\mathrm{COH}$ cycles & 87 & 105 & \\
\hline Number of $\mathrm{COH}$ cycles per patient & 1.04 & 1.18 & 0.007 \\
\hline Gonadotropin days $(\overline{\mathrm{x}} \pm \mathrm{SD})$ & $10.2 \pm 2.1$ & $10.5 \pm 2.9$ & 0.416 \\
\hline Gonadotropin dosage $(\times 75 \mathrm{IU}, \overline{\mathrm{x}} \pm \mathrm{SD})$ & $2,065.3 \pm 768.1$ & $1,956.3 \pm 856.9$ & 0.359 \\
\hline Number of retrieval oocytes $(\overline{\mathrm{x}} \pm \mathrm{SD})$ & $12.8 \pm 7.0$ & $12.8 \pm 7.3$ & 0.977 \\
\hline Number of cleaving zygotes $(\overline{\mathrm{x}} \pm \mathrm{SD})$ & $8.9 \pm 4.7$ & $9.5 \pm 6.0$ & 0.441 \\
\hline Number of blastocysts & $4.7 \pm 3.3$ & $4.4 \pm 3.1$ & 0.513 \\
\hline Number of FET cycles & 113 & 136 & \\
\hline Embryos at d 5 & $80(70.8 \%)$ & $114(83.8 \%)$ & 0.014 \\
\hline Embryos at $d 6$ & $33(29.2 \%)$ & $22(16.2 \%)$ & \\
\hline Blastocyst quality, Good (AA/BA/AB) & $36(31.9 \%)$ & $45(33.1 \%)$ & 0.837 \\
\hline Blastocyst quality, Fair (BB/AC) & $35(31.0 \%)$ & $58(42.6 \%)$ & 0.058 \\
\hline Blastocyst quality, Poor (CA/BC/CB) & $42(37.2 \%)$ & $33(24.3 \%)$ & 0.027 \\
\hline
\end{tabular}


$\mathrm{COH}$, controlled ovarian hyperstimulation; SD, standard deviation; FET, frozen-thawed embryo transfer.

Table 2. Baseline demographics of patients with recurrent implantation failure (RIF) after embryo selection by non-invasive chromosome screening (NICS) versus blastocyst morphology (Non-NICS). 


\begin{tabular}{|c|c|c|c|}
\hline Variable & NICS & Non-NICS & $p$-value \\
\hline Number of patients & 44 & 56 & \\
\hline Female age $(y)$ & $32.1 \pm 4.7$ & $33.3 \pm 4.3$ & 0.143 \\
\hline Female body mass index $\left(\mathrm{kg} \mathrm{m}^{-2}\right)$ & $21.0 \pm 2.5$ & $21.7 \pm 2.4$ & 0.101 \\
\hline Male age $(y)$ & $34.2 \pm 5.2$ & $34.9 \pm 4.9$ & 0.407 \\
\hline Male body mass index $\left(\mathrm{kg} \mathrm{m}^{-2}\right)$ & $24.2 \pm 3.4$ & $23.8 \pm 3.4$ & 0.498 \\
\hline Infertility duration (y) & $3.5 \pm 2.9$ & $5.2 \pm 3.0$ & 0.004 \\
\hline Number of prior miscarriages & $1.9 \pm 1.2$ & $0.8 \pm 1.1$ & $<0.001$ \\
\hline Number of previous embryo transfers & $4.4 \pm 1.3$ & $4.2 \pm 1.3$ & 0.379 \\
\hline Number of prior live births & $1 / 44(2.3 \%)$ & 0 & \\
\hline Prolactin $\left(\mathrm{ng} \mathrm{m}^{-1}\right)$ & $26.2 \pm 76.4$ & $15.1 \pm 9.9$ & 0.231 \\
\hline Testosterone & $0.5 \pm 0.2$ & $0.5 \pm 0.3$ & 0.973 \\
\hline Progesterone & $0.8 \pm 1.0$ & $0.6 \pm 0.3$ & 0.287 \\
\hline Follicle-stimulating hormone & $7.8 \pm 2.4$ & $7.5 \pm 3.5$ & 0.608 \\
\hline Luteinizing hormone & $5.2 \pm 2.2$ & $4.5 \pm 2.7$ & 0.108 \\
\hline Estradiol & $50.4 \pm 25.8$ & $51.2 \pm 41.70$ & 0.913 \\
\hline Number of $\mathrm{COH}$ cycles & 44 & 70 & \\
\hline Number of $\mathrm{COH}$ cycles per patient & 1 & 1.3 & $<0.001$ \\
\hline Gonadotropin days $(\overline{\mathrm{x}} \pm \mathrm{SD})$ & $10.0 \pm 2.4$ & $11.0 \pm 2.4$ & 0.03 \\
\hline Gonadotropin dosage $(\times 75 \mathrm{IU}, \overline{\mathrm{x}} \pm \mathrm{SD})$ & $1,838.4 \pm 748.3$ & $2,192.1 \pm 872.7$ & 0.028 \\
\hline Number of retrieval oocytes $(\overline{\mathrm{x}} \pm \mathrm{SD})$ & $13.9 \pm 7.5$ & $13.0 \pm 7.5$ & 0.551 \\
\hline Number of cleaving zygotes $(\overline{\mathrm{x}} \pm \mathrm{SD})$ & $10.8 \pm 6.1$ & $9.7 \pm 5.4$ & 0.319 \\
\hline Number of blastocysts $(\overline{\mathrm{x}} \pm \mathrm{SD})$ & $5.3 \pm 2.8$ & $4.2 \pm 3.2$ & 0.059 \\
\hline Number of FET cycles & 64 & 94 & \\
\hline Embryos at d 5 & $48(75.0 \%)$ & $64(68.1 \%)$ & 0.24 \\
\hline Embryos at $d 6$ & $16(25.0 \%)$ & $30(31.9 \%)$ & \\
\hline Blastocyst quality, Good (AA/BA/AB) & $11(17.2 \%)$ & $22(23.4 \%)$ & 0.345 \\
\hline Blastocyst quality, Fair (BB/AC) & $31(48.4 \%)$ & $34(36.2 \%)$ & 0.124 \\
\hline Blastocyst quality, Poor (CA/BC/CB) & $22(34.4 \%)$ & $38(40.4 \%)$ & 0.442 \\
\hline
\end{tabular}


$\mathrm{COH}$, controlled ovarian hyperstimulation; SD, standard deviation; FET, frozen-thawed embryo transfer.

Data variables analyzed in this study were age at retrieval, the history of pregnancies, live births, and miscarriages, BMI, hormone levels, the number of oocyte retrieval cycles, oocytes, cleavages, and D5 or D6 blastocysts, and blastocyst morphology (expansion, inner cell mass, and trophectoderm) were comparable by Student's t test or Mann-Whitney $U$ test, each as appropriate (Tables 1 and 2 ).

Our analysis showed no significant differences in age, BMI and hormone levels between the NICS and non-NICS groups in RPL or RIF patients. The number of previous miscarriages in the NICS group of patients with RPL or RIF was significantly higher than the non-NICS group. Besides, infertility duration showed statistical significance $(p=0.001, p=0.004)$ in the NICS group and non-NICS group no matter which feature the patients are in. The number of miscarriages and infertility duration had impact on clinical outcomes. Thus, they were included in the multiple logistic regression analysis.

\section{Blastocyst Morphological Assessments and NICS results}

The blastocyst transplantation was based on morphology and NICS results. Blastocysts were evaluated based on their development and quality using the Gardner scores system and divided into three categories: good (AA/BA/AB), fair (BB/AC), and poor (CA/BC/CB) [31]. As shown in Tables 1 and 2, no significant differences were observed between NICS group and control group of patients with RIF. Of the patients with RPL the non-NICS group was lower than NICS group (37.2\% vs. $24.3 \%$ ) in the poor category, however, there was no significant difference between the other two categories.

In the NICS assay, we sequenced approximately 2 million reads on each culture medium sample. The read numbers were counted along the 24 chromosomes with a bin size of $1 \mathrm{Mb}$ and normalized by the mean of the corresponding bin in all samples. Examples of the results of NICS were demonstrated in Additional File 1.

\section{Comparison of clinical outcomes between NICS and non-NICS Groups for Patients with RPL or RIF}

For the RPL patients, the miscarriage rate per FET was significantly lower in the NICS group than in the non-NICS group ( $17.9 \%$ vs. $42.6 \%$, adjusted OR $0.39,95 \% \mathrm{Cl} 0.16-0.95)$, whereas the ongoing pregnancy rate $(40.7 \%$ vs. $25.0 \%$, adjusted OR $2.0,95 \% \mathrm{Cl} 1.04-3.82)$ and live birth rate $(38.9 \%$ vs. $20.6 \%$, adjusted OR $2.53,95 \% \mathrm{Cl} 1.28-5.02$ ) were significantly higher in the NICS group compared to the non-NICS group (Fig. 2; Table 3). Nevertheless, no differences were identified in pregnancy rates per patient between the NICS and non-NICS groups (49.6\% vs. $44.9 \%$, adjusted OR 1.13, 95\% Cl 0.61-2.07; Table 3).

Table 3. Comparison of clinical outcomes patients with recurrent pregnancy loss (RPL). 


\begin{tabular}{|c|c|c|c|}
\hline & NICS & Non-NICS & $\begin{array}{l}\text { Adjusted odds ratio }(95 \% \mathrm{Cl}), \mathrm{p}- \\
\text { value }\end{array}$ \\
\hline $\begin{array}{l}\text { Number of transferred } \\
\text { cycles }\end{array}$ & 113 & 136 & \\
\hline $\begin{array}{l}\text { Clinical pregnancies rate } \\
(\%)\end{array}$ & $\begin{array}{l}56 / 113 \\
(49.6 \%)\end{array}$ & $\begin{array}{l}61 / 136 \\
(44.9 \%)\end{array}$ & $1.13(0.61-2.07), 0.697$ \\
\hline Miscarriages rate (\%) & $10 / 56(17.9 \%)$ & $26 / 61(42.6 \%)$ & $0.39(0.16-0.95), 0.038$ \\
\hline Ectopic pregnancies & 0 & $1 / 61(1.6 \%)$ & \\
\hline $\begin{array}{l}\text { Ongoing pregnancies rate } \\
(\%)\end{array}$ & $\begin{array}{l}46 / 113 \\
(40.7 \%)\end{array}$ & $\begin{array}{l}34 / 136 \\
(25.0 \%)\end{array}$ & $2.0(1.04-3.82), 0.037$ \\
\hline Live birth rate (\%) & $\begin{array}{l}44 / 113 \\
(38.9 \%)\end{array}$ & $\begin{array}{l}28 / 136 \\
(20.6 \%)\end{array}$ & $2.53(1.28-5.02), 0.008$ \\
\hline \multicolumn{4}{|c|}{$\begin{array}{l}\text { NICS group, embryo selection by non-invasive chromosome screening; control group, blastocyst } \\
\text { morphology. }\end{array}$} \\
\hline \multicolumn{4}{|c|}{$\begin{array}{l}\text { *Adjusted for female age, infertility duration, number of prior miscarriages, number of previous } \\
\text { embryo transfers, number of } \mathrm{COH} \text { cycles, embryo morphology and Day } 5 / 6 \text { blastocysts }\end{array}$} \\
\hline
\end{tabular}

For the RIF patients, the pregnancy rates per FET were significantly higher in the NICS group than in the non-NICS group (46.9\% vs. $28.7 \%$, adjusted OR 2.82 95\% Cl 1.20-6.66. Fig. 3; Table 4), whereas the live birth rate and ongoing pregnancy rate per FET and per patient were higher in the NICS group than in the non-NICS group, but not significantly. Nevertheless, no differences were identified in the miscarriage rate per clinical pregnancy between the NICS and non-NICS groups $(23.3 \%$ vs. $25.9 \%$, adjusted OR $1.35,95 \% \mathrm{CI}$ 0.34-5.42; Table 4).

Table 4. Comparison of clinical outcomes patients with recurrent implantation failure (RIF). 


\begin{tabular}{|c|c|c|c|}
\hline & NICS & Non-NICS & $\begin{array}{l}\text { Adjusted odds ratio }(95 \% \mathrm{Cl}) \text {, p- } \\
\text { value }\end{array}$ \\
\hline $\begin{array}{l}\text { Number of transferred } \\
\text { cycles }\end{array}$ & 64 & 94 & \\
\hline Clinical pregnancies rate (\%) & $\begin{array}{l}30 / 64 \\
(46.9 \%)\end{array}$ & $\begin{array}{l}27 / 94 \\
(28.7 \%)\end{array}$ & $2.82(1.20-6.66), 0.018$ \\
\hline Miscarriages rate (\%) & $7 / 30(23.3 \%)$ & $7 / 27(25.9 \%)$ & $1.35(0.34-5.42), 0.671$ \\
\hline Ectopic pregnancies & $3 / 30(10 \%)$ & 0 & \\
\hline $\begin{array}{l}\text { Ongoing pregnancies rate } \\
(\%)\end{array}$ & $\begin{array}{l}20 / 64 \\
(31.3 \%)\end{array}$ & $\begin{array}{l}20 / 94 \\
(21.3 \%)\end{array}$ & $2.19(0.88-5.47), 0.094$ \\
\hline Live birth rate (\%) & $\begin{array}{l}19 / 64 \\
(29.7 \%)\end{array}$ & $\begin{array}{l}20 / 94 \\
(21.3 \%)\end{array}$ & $1.96(0.78-4.92), 0.154$ \\
\hline \multicolumn{4}{|c|}{$\begin{array}{l}\text { NICS group, embryo selection by non-invasive chromosome screening; control group, blastocyst } \\
\text { morphology. }\end{array}$} \\
\hline \multicolumn{4}{|c|}{$\begin{array}{l}\text { *Adjusted for female age, infertility duration, number of prior miscarriages, number of } \mathrm{COH} \text { cycles, } \\
\text { gonadotropin days, gonadotropin dosage, number of blastocysts embryo morphology and Day } 5 / 6 \\
\text { blastocysts }\end{array}$} \\
\hline
\end{tabular}

\section{Discussion}

To our knowledge, this is the first large-scale study to confirm the effectiveness of NICS as a diagnostic test for the outcomes of IVF in women with a history of RPL or RIF. We demonstrated that the miscarriage rate was significantly lower in women with RPL $(n=173)$, whereas the pregnancy rate was significantly higher in the NICS group than in the non-NICS group of women with RIF $(n=100)$. To confirm that the improvement of clinical outcomes in the NICS group was related to the genetic testing alone irrespective of the other factors, we included the variables at $p<0.10$ and related to the clinical outcome in the adjusted models. Our results strongly supported that the high clinical value of NICS in women with RPL prior to embryo transfer since it significantly increased the live birth rate per transfer cycle through the multiple logistic regression analysis ( $38.9 \%$ vs. $20.6 \%$, adjusted OR $2.46,95 \% \mathrm{Cl} 1.41-4.35)$. The pregnancy rates per FET in the NICS group were significantly higher than in the non-NICS control group (46.9\% vs. $28.7 \%$, adjusted OR $2.82,95 \% \mathrm{Cl} 1.20-6.66)$ for the PIF patients. Similarly, Fang et al. obtained an ongoing pregnancy rate of $58 \%$ and reported 27 normal live births in patients with RPL or RIF after transferring 50 embryos identified as euploid by NICS [28].

In the present study, women with RPL had a history of $\geq 2$ miscarriages, spontaneous abortion and/or biochemical pregnancy[30,31]. The definition of RPL differs among organizations. For instance, the Royal College of Obstetricians and Gynecologists and the European Society of Human Reproduction and Embryology support that a miscarriage includes all pregnancy losses up to 24 weeks of gestation, whereas the American Society for Reproductive Medicine does not provide a time limit and excludes biochemical pregnancy[32]. Thus, there is no consensus on whether biochemical pregnancy should be 
considered RPL. It is known that the rate of chromosome abnormality decreases with the developmental stage: $70-80 \%$ in clinical miscarriages[33], $4 \%$ in stillbirths, and $0.3 \%$ in newborn babies [34]. The selection of euploid embryos can reduce the rate of miscarriages, which are probably caused by chromosome abnormalities[35].

Women with RIF had a history of $\geq 3$ failed implantations of $4-6$ high-score blastomeres or $>3$ highscore blastocysts. A retrospective analysis of 10,711 blastocysts showed that the incidence of blastocyst aneuploidy in RIF patients is significantly higher than that in the control group, and embryo aneuploidy is a primary reason for implantation failure [6]. A clinical study suggested that transplanting euploid embryos may help patients with RIF to achieve pregnancy[36]. A multi-center, prospective, pilot study showed that screening for embryo ploidy improved the live birth rate per embryo transfer procedure in both RPL (52.4\% vs $21.6 \%$ ) and RIF group (62.5\% vs $31.7 \%)[37]$.

Chromosome aneuploidy is common in embryos following IVF, even in younger women, and is a major failure factor. Although morphology is clearly correlated with euploidy, it can only increase euploidy by a few percentage points at most when used to select replacement embryos. Embryos with good morphology may have chromosomal abnormalities [9]. Routine embryo screening for aneuploidy requires intensive biopsy procedures, which are costly and time-consuming. The accuracy of NICS has been verified in numerous studies, and a $58 \%$ clinical pregnancy success rate has been reported for patients with RIF and RPL [28]. Recently, the utility of niPGT-A is challenged in the article by Hanson et al. [38]. Here, we also found that NICS allowed the selection of euploid embryos, increasing the live birth rates in women with RPL and improve the clinical pregnancy rates in women with RIF based on many patients, which is meaningful to both clinicians and basic scientists on NICS assay. Our center is currently participating in a multi-unit clinical trial for women $\geq 35$ years of age to further validate the clinical value of NICS since a more comprehensive application requires advanced technology such as modified wholegenome amplification and sequencing protocols as well as novel diagnostic algorithms. The validation of NICS in different populations and the accumulation of detectable sample sizes may help markedly reduce the detection cost per embryo.

There are some limitations in our study: 1) as a retrospective study, patients in the NICS groups had different clinical prognoses than those in the non-NICS groups, introducing bias in the study results, and 2) the loss and cycle cancellation caused by euploid selection after NICS was not calculated since only women with a good prognosis reach the blastocyst stage and have a euploid embryo to transfer.

\section{Conclusions}

Our large-scale retrospective study demonstrated noninvasive chromosome screening for aneuploidy improved the clinical outcomes for patients with recurrent pregnancy loss or repeated implantation failure. NICS could be used as a diagnostic test in clinical practice.

\section{List Of Abbreviations}


NICS noninvasive chromosome screening

RPL recurrent pregnancy loss

RIF repeated implantation failure

FET frozen embryo transfer

IVF-ET in vitro fertilization-embryo transfer

TE trophectoderm

BMI body mass index

hCG human chorionic gonadotropin

WGA Whole-genome amplification

CBS circular binary segmentation

\section{Declarations}

\section{Declaration of Interests}

Authors declare no conflicts of interests.

\section{Acknowledgments}

The authors would like to thank Shiping Bo and Shujie Ma for their assistance in NGS data analysis as well as Tuan Li and Yangyun Zou for statistical analysis.

\section{Funding}

This study was supported by the Research Fund for Lin He's Academician Workstation of New Medicine and Clinical Translation at the Second Affiliated Hospital of Wenzhou Medical University (Grant no. 18331105) and the National Key Research and Development Program (Grant no. 2018YFC1003103)

\section{Ethics approval and consent to participate}

This study was approved by the Institutional Review Board (IRB) of the Second Affiliated Hospital of Wenzhou Medical University (number: 2021-K-13-01). Informed consents were obtained in accordance with the institutional guidelines prior to embryo analysis.

\section{Consent for publication}

All authors read and approved the final manuscript. 


\section{Availability of data and materials}

The original contributions presented in the study are included in the article/Supplementary Material.

\section{References}

1. Gardner DK, Schoolcraft WB. Culture and transfer of human blastocysts. Curr Opin Obstet Gynecol. 1999;11:307-11.

2. Alfarawati S, Fragouli E, Colls P, Stevens J, Gutierrez-Mateo C, Schoolcraft WB, et al. The relationship between blastocyst morphology, chromosomal abnormality, and embryo gender. Fertil Steril. 2011;95:520-4.

3. Capalbo A, Rienzi L, Cimadomo D, Maggiulli R, Elliott T, Wright G, et al. Correlation between standard blastocyst morphology, euploidy and implantation: an observational study in two centers involving 956 screened blastocysts. Hum Reprod. 2014;29:1173-81.

4. Dai R, Xi Q, Wang R, Zhang H, Jiang Y, Li L, et al. Chromosomal copy number variations in products of conception from spontaneous abortion by next-generation sequencing technology. Medicine (Baltimore) 2019;98:e18041.

5. Elkarhat Z, Kindil Z, Zarouf L, Razoki L, Aboulfaraj J, Elbakay C, et al. Chromosomal abnormalities in couples with recurrent spontaneous miscarriage: a 21-year retrospective study, a report of a novel insertion, and a literature review. J Assist Reprod Genet. 2019;36:499-507.

6. Kort JD, McCoy RC, Demko Z, Lathi RB. Are blastocyst aneuploidy rates different between fertile and infertile populations? J Assist Reprod Genet. 2018;35:403-8.

7. Practice Committee of the American Society for Reproductive M. Definitions of infertility and recurrent pregnancy loss: a committee opinion. Fertil Steril. 2013;99:63.

8. Sahoo T, Dzidic N, Strecker MN, Commander S, Travis MK, Doherty C, et al. Comprehensive genetic analysis of pregnancy loss by chromosomal microarrays: outcomes, benefits, and challenges. Genet Med. 2017;19:83-9.

9. Munné S, Wells $D$, Cohen J. Technology requirements for preimplantation genetic diagnosis to improve assisted reproduction outcomes. Fertil Steril. 2010;94:408-30.

10. Greco E, Bono S, Ruberti A, Lobascio AM, Greco P, Biricik A, et al. Comparative genomic hybridization selection of blastocysts for repeated implantation failure treatment: a pilot study. Biomed Res Int. 2014;2014:457913.

11. Lei C-X, Ye J-F, Sui Y-L, Zhang Y-P, Sun X-XJR, Medicine D. Retrospective cohort study of preimplantation genetic testing for aneuploidy with comprehensive chromosome screening versus nonpreimplantation genetic testing in normal karyotype, secondary infertility patients with recurrent pregnancy loss 2019;3:205.

12. Cozzolino M, Diaz-Gimeno P, Pellicer A, Garrido N. Evaluation of the endometrial receptivity assay and the preimplantation genetic test for aneuploidy in overcoming recurrent implantation failure. $J$ Assist Reprod Genet. 2020;37:2989-97. 
13. Bhatt SJ, Marchetto NM, Roy J, Morelli SS, McGovern PG. Pregnancy outcomes following in vitro fertilization frozen embryo transfer (IVF-FET) with or without preimplantation genetic testing for aneuploidy (PGT-A) in women with recurrent pregnancy loss (RPL): a SART-CORS study. Hum Reprod. 2021;36:2339-44.

14. Lee $\mathrm{Cl}$, Wu CH, Pai YP, Chang YJ, Chen $\mathrm{Cl}$, Lee TH, Lee MS. Performance of preimplantation genetic testing for aneuploidy in IVF cycles for patients with advanced maternal age, repeat implantation failure, and idiopathic recurrent miscarriage. Taiwan J Obstet Gynecol. 2019;58:239-43.

15. Grati FR, Gallazzi G, Branca L, Maggi F, Simoni G, Yaron Y. An evidence-based scoring system for prioritizing mosaic aneuploid embryos following preimplantation genetic screening. Reprod Biomed Online 2018;36:442-9.

16. Hodes-Wertz B, Grifo J, Ghadir S, Kaplan B, Laskin CA, Glassner M, et al. Idiopathic recurrent miscarriage is caused mostly by aneuploid embryos. Fertil Steril. 2012;98:675-80.

17. Zeng Y, Lv Z, Gu L, Wang L, Zhou Z, Zhu H et al. Preimplantation genetic diagnosis (PGD) influences adrenal development and response to cold stress in resulting mice. Cell Tissue Res. 2013;354:72941.

18. Zhao HC, Zhao Y, Li M, Yan J, Li L, Li R, et al. Aberrant epigenetic modification in murine brain tissues of offspring from preimplantation genetic diagnosis blastomere biopsies. Biol Reprod. 2013;89:117.

19. Middelburg KJ, Heineman MJ, Haadsma ML, Bos AF, Kok JH, Hadders-Algra M. Neurological condition of infants born after in vitro fertilization with preimplantation genetic screening. Pediatr Res. 2010;67:430-4.

20. Zhang S, Luo K, Cheng D, Tan Y, Lu C, He H, et al. Number of biopsied trophectoderm cells is likely to affect the implantation potential of blastocysts with poor trophectoderm quality. Fertil Steril. 2016;105:1222-7 e4.

21. Guzman L, Nunez D, Lopez R, Inoue N, Portella J, Vizcarra F, et al. The number of biopsied trophectoderm cells may affect pregnancy outcomes. J Assist Reprod Genet. 2019;36:145-51.

22. Stigliani S, Anserini P, Venturini PL, Scaruffi P. Mitochondrial DNA content in embryo culture medium is significantly associated with human embryo fragmentation. Hum Reprod. 2013;28:2652-60.

23. Yeung QSY, Zhang YX, Chung JPW, Lui WT, Kwok YKY, Gui B, et al. A prospective study of noninvasive preimplantation genetic testing for aneuploidies (NiPGT-A) using next-generation sequencing (NGS) on spent culture media (SCM). J Assist Reprod Genet. 2019;36:1609-21.

24. Shamonki MI, Jin H, Haimowitz Z, Liu L. Proof of concept: preimplantation genetic screening without embryo biopsy through analysis of cell-free DNA in spent embryo culture media. Fertil Steril. 2016;106:1312-8.

25. Rubio C, Navarro-Sanchez L, Garcia-Pascual CM, Ocali O, Cimadomo D, Venier W, et al. Multicenter prospective study of concordance between embryonic cell-free DNA and trophectoderm biopsies from 1301 human blastocysts. Am J Obstet Gynecol. 2020;223:751 e1-13.

26. Xu J, Fang R, Chen L, Chen D, Xiao JP, Yang W, et al. Noninvasive chromosome screening of human embryos by genome sequencing of embryo culture medium for in vitro fertilization. Proc Natl Acad 
Sci U S A. 2016;113:11907-12.

27. Huang L, Bogale B, Tang Y, Lu S, Xie XS, Racowsky C. Noninvasive preimplantation genetic testing for aneuploidy in spent medium may be more reliable than trophectoderm biopsy. Proc Natl Acad Sci U S A. 2019;116:14105-12.

28. Fang R, Yang W, Zhao X, Xiong F, Guo C, Xiao J, et al. Chromosome screening using culture medium of embryos fertilised in vitro: a pilot clinical study. J Transl Med. 2019;17:73.

29. Munné S, Kaplan B, Frattarelli JL, Child T, Nakhuda G, Shamma FN, et al. Preimplantation genetic testing for aneuploidy versus morphology as selection criteria for single frozen-thawed embryo transfer in good-prognosis patients: a multicenter randomized clinical trial. Fertil Steril. 2019;112:1071-9.e7.

30. No RG-tGJRL, UK. The investigation and treatment of couples with recurrent first-trimester and second-trimester miscarriage 2011.

31. RPL EGGo, Bender Atik R, Christiansen OB, Elson J, Kolte AM, Lewis S et al. ESHRE guideline: recurrent pregnancy loss. Hum Reprod Open 2018;2018:hoy004.

32. Practice Committee of the American Society for Reproductive M. Evaluation and treatment of recurrent pregnancy loss: a committee opinion. Fertil Steril 2012;98:1103-11.

33. Ogasawara M, Aoki K, Okada S, Suzumori K. Embryonic karyotype of abortuses in relation to the number of previous miscarriages. Fertil Steril 2000;73:300-4.

34. Nagaoka SI, Hassold TJ, Hunt PAJNRG. Human aneuploidy: mechanisms and new insights into an age-old problem 2012;13:493-504.

35. Forman EJ, Hong KH, Franasiak JM, Scott RT, Jr. Obstetrical and neonatal outcomes from the BEST Trial: single embryo transfer with aneuploidy screening improves outcomes after in vitro fertilization without compromising delivery rates. Am J Obstet Gynecol 2014;210:157 e1-6.

36. McCoy RC, Demko ZP, Ryan A, Banjevic M, Hill M, Sigurjonsson S et al. Evidence of Selection against Complex Mitotic-Origin Aneuploidy during Preimplantation Development. PLoS Genet 2015;11:e1005601.

37. Sato T, Sugiura-Ogasawara M, Ozawa F, Yamamoto T, Kato T. Preimplantation genetic testing for aneuploidy: a comparison of live birth rates in patients with recurrent pregnancy loss due to embryonic aneuploidy or recurrent implantation failure. Hum Reprod. 2019;34:2340-8.

38. Hanson BM, Tao X, Hong KH, Comito CE, Pangasnan R, Seli E et al. Noninvasive preimplantation genetic testing for aneuploidy exhibits high rates of deoxyribonucleic acid amplification failure and poor correlation with results obtained using trophectoderm biopsy. Fertil Steril 2021;115:1461-70.

\section{Figures}




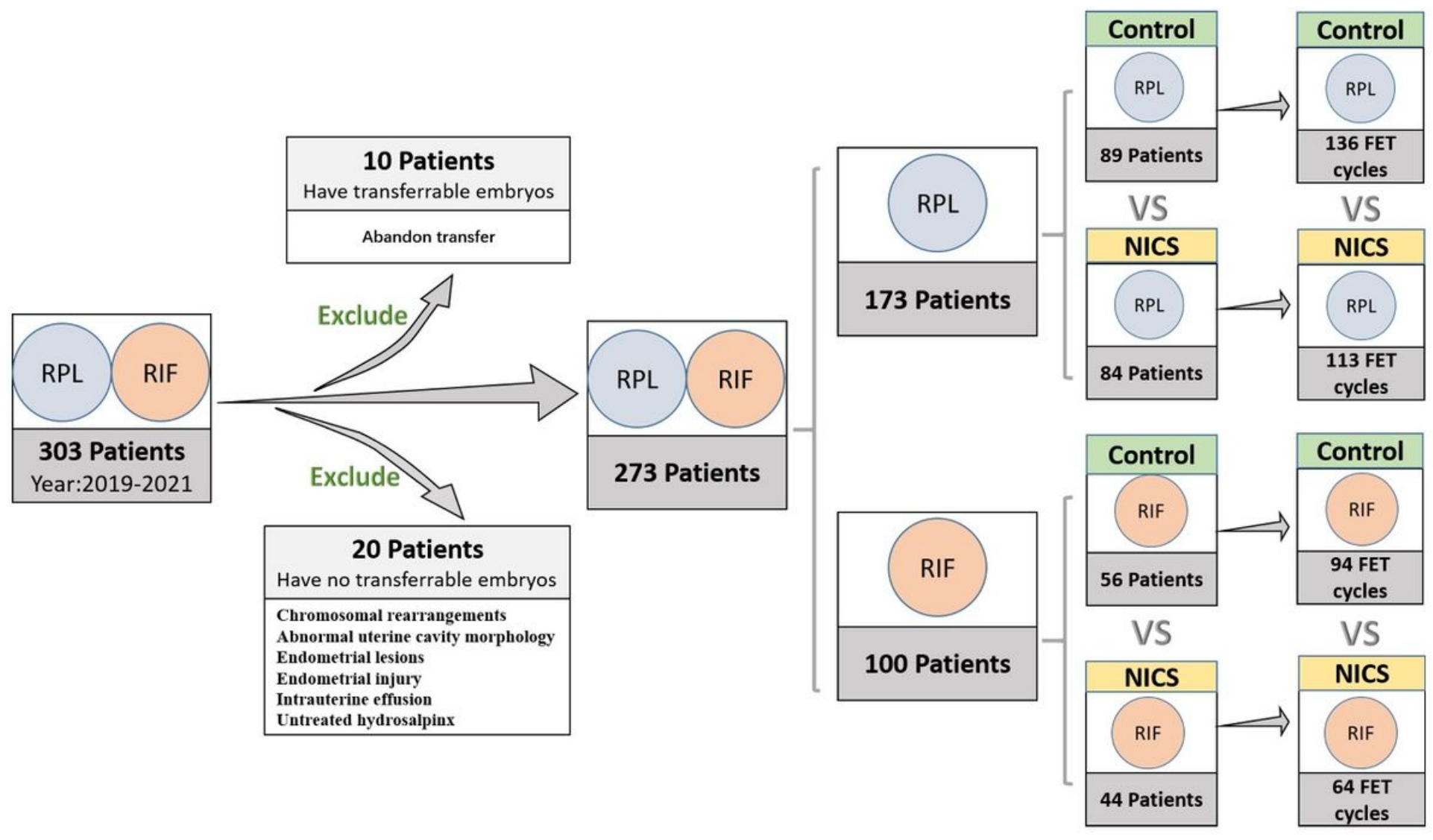

\section{Figure 1}

Inclusion and classification of patient selection in this study. NICS, non-invasive chromosome screening; RPL, recurrent pregnancy loss; RIF, repeated implantation failure; Control, blastocyst morphology (nonNICS). 


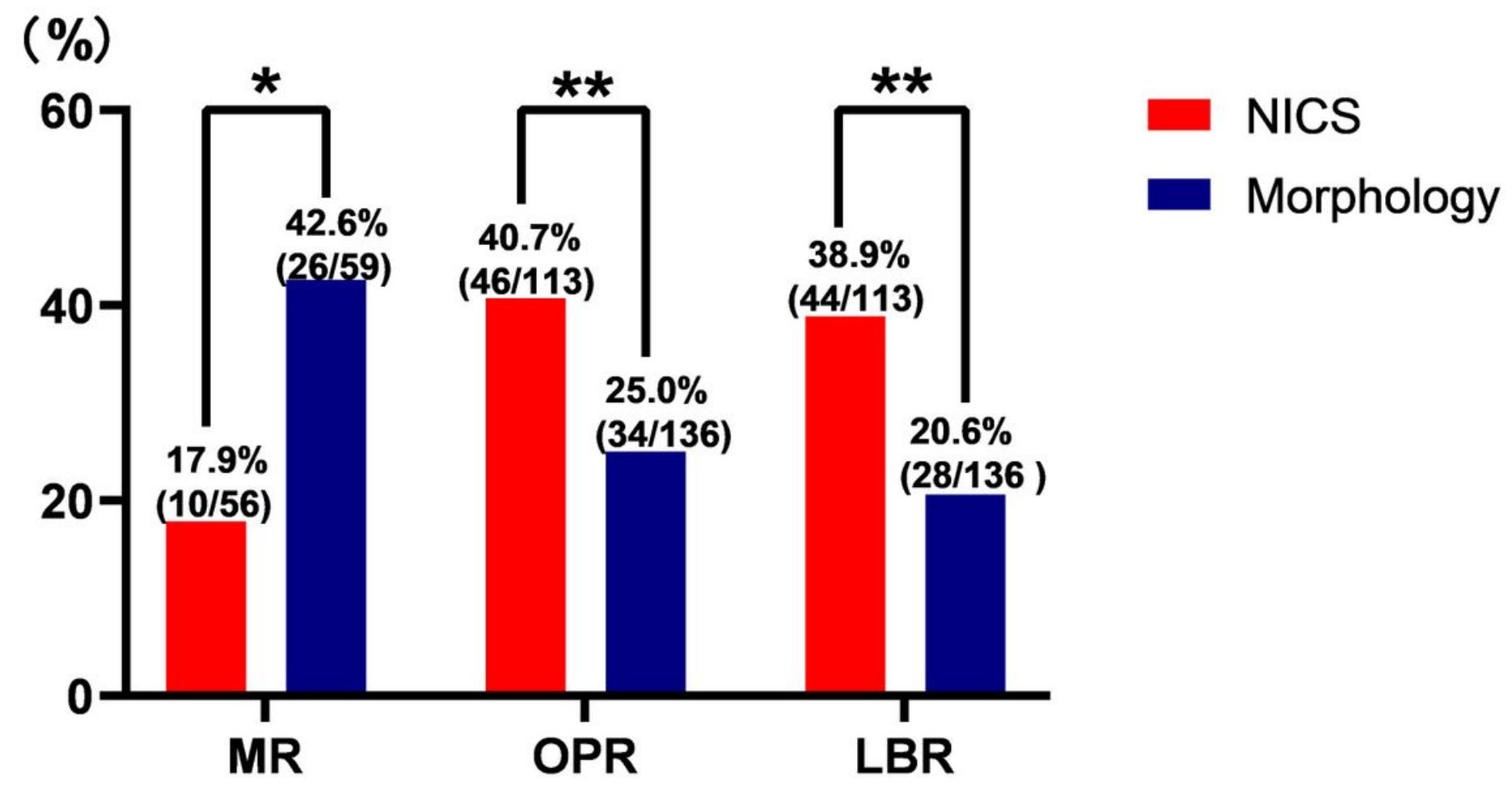

Figure 2

Pregnancy outcomes of patients with recurrent pregnancy loss (RPR) after embryo selection by noninvasive chromosome screening (NICS) versus blastocyst morphology (control). MR, miscarriages rate; OPR, ongoing pregnancy rate; LBR, live birth rate. ${ }^{\star} p<0.05,{ }^{\star} * \mathrm{p}<0.01$. 


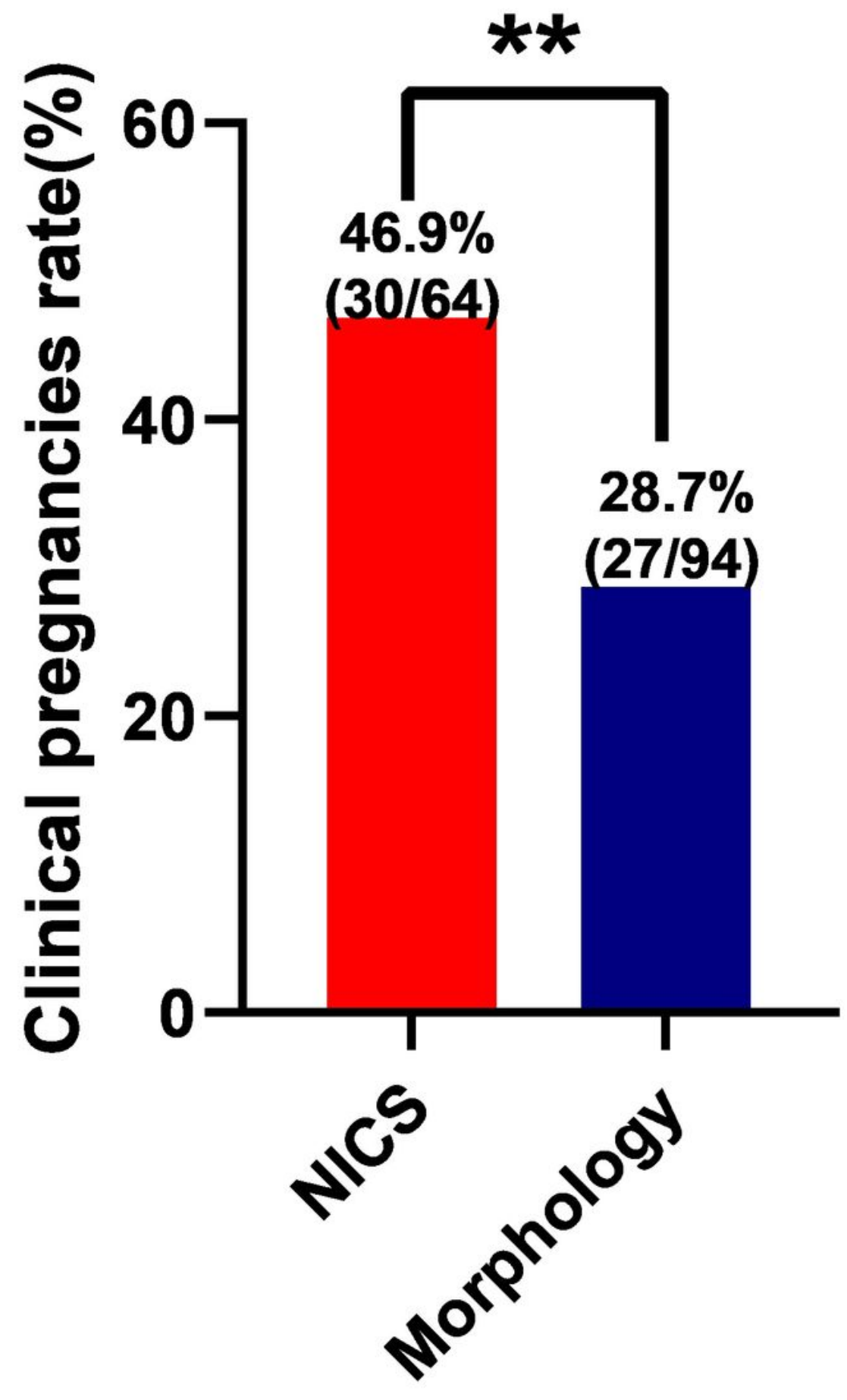

Figure 3

Clinical pregnancy rate of patients repeated implantation failure (RIF) after embryo selection by noninvasive chromosome screening (NICS) versus blastocyst morphology (control). ${ }^{\star} p<0.05$.

\section{Supplementary Files}


This is a list of supplementary files associated with this preprint. Click to download.

- AdditionalFile.docx 